Gdańsk 2019, Nr. 41

https://doi.org/10.26881/sgg.2019.41.12

Viktor Tichák

Karls-Universität in Prag

https://orcid.org/0000-0001-7842-9956

\title{
Die Charaktere des Erfolgs aus linguistischer Sicht ${ }^{1}$
}

Die Studie präsentiert ein neu definiertes System der Kategorien Aspektualität, Aktionsart und Verbalcharakter. Das Ziel ist es, die Existenz semantisch markierter tschechischer Verben zu beschreiben, die die letzte Phase eines durativen Prozesses und dessen erfolgreichen Abschluss ausdrücken (z.B. dockat se und čekat) und die semantische Kategorie ,Erfolg' im Tschechischen und Deutschen systematisch einzuordnen.

Schlüsselwörter: Aspektualität, Aspekt, Aktionsart, Verbalcharakter, Erfolg.

The Types of Success from a Linguistic Point of View. - The article presents a newly defined system of the categories aspectuality, aspect, aktionsart and verb type. The aim is to explain the existence of semantically marked Czech verbs which express the last phase of a durative process and its successful completion (e.g. dockat se and crekat) and to map the place of the semantical category 'success' in the aspectological system of the Czech and German language.

Keywords: aspectuality, aspect, aktionsart, verb type, success.

\section{Einleitung}

Erfolg ist ein „positives Ergebnis einer Bemühung; Eintreten einer beabsichtigten, erstrebten Wirkung," so die Duden-Definition. ${ }^{2}$ Der Terminus bezeichnet also einen Zeitpunkt, präsupponiert zugleich aber eine Bemühung genau um das Erreichen des erwünschten Punkts. Es stellt sich dann die Gegenfrage: Kann Erfolg ohne Bemühung eintreten? Wäre etwa ein Gewinn in der Lotterie ein Erfolg, wenn der Gewinner den Tippschein erst nach dem Gewinn geschenkt bekäme? Wird ein Verb wie finden oder gewinnen immer von einem Suchen bzw. Spielen antizipiert, was im Beispiel (2) als fraglich scheint?

(1) Ich habe die zweite Socke endlich gefunden.

(2) Ich habe auf der Straße eine Hundertnote gefunden.

1 Die Studie ist mit Unterstützung von MŠMT entstanden (Grant IGA_FF_2018_034).

2 URL: https://www.duden.de/rechtschreibung/Erfolg (20.10.2018). 
Die Aspektologen des 20. und 21. Jahrhunderts arbeiten mit Erfolgsverben in ihren systematischen Beschreibungen der Einzelsprachen (wie VENDLER 1957, SCHOpF 1984, TSCHIRNER 1991 u.a.) oder in den Versuchen um eine nicht-sprachspezifische Beschreibung des Systems interner Zeitstrukturierung der Verben oder Prädikate (wie ComrIe 1981). Die größten Schwierigkeiten in allen Beschreibungen stellen die folgenden drei Punkte dar: (A) die Terminologie, die reich und uneinheitlich ist; (B) die verschiedenen Sprachen und damit verschiedenen Systeme, vor allem dann die Vorherrschaft der englischsprachigen Quellen, die primär mit der englischen Sprache arbeiten; (C) die unterschiedlichen Ausprägungen der Aspektualität, die morphologischer, (morpho)syntaktischer und semantischer Natur sind.

Die Ausgangssprachen der folgenden Arbeit sind Tschechisch und Deutsch. Der Vergleich eines slawischen und eines germanischen Sprachsystems zeigt bei der Analyse des Erfolgs viele Unterschiede, z.B.:

(3) Ich habe meine Dissertation verteidigt.

(3a) Obhájil jsem svou disertaci. / (3b) Obhajoval jsem svou disertaci.

Der deutsche Satz hat nur eine Interpretation, nämlich die resultative/erfolgreiche: Die Arbeit ist jetzt verteidigt. Der tschechische Satz kann mit dem morphologischen Aspekt spielen und damit entweder eine perfektive oder eine imperfektive Lesart erschaffen. Um die 3b-Situation im Deutschen zu beschreiben, braucht man andere Mittel, wie in (3c).

(3c) Die Verteidigung meiner Dissertation hat stattgefunden.

Außerdem gibt es im Tschechischen eine Gruppe reflexiver Verben, die systematisch mit dem Präfix do- gebildet werden und den erfolgreichen Abschluss der Basisverb-Prädikation ausdrücken, z.B. dočkat se, doklepatse, dovolat se, dozvonit se. Ich führe keine deutschen Äquivalente an, denn das Deutsche verfügt über solche Verben nicht und eine Situation wie in (4) ist nicht wortwörtlich zu übersetzen.

(4) A: Čekal jsem dvě hodiny. B: A dočkal ses?

(4a) A: Ich habe zwei Stunden gewartet. B: Und erfolgreich?

Die Erfolgskomponente wird in der tschechisch-deutschen Lexikographie oft nur unzulänglich bzw. in irreführender Weise erfasst, indem das Verb dockat se lediglich mit ,erwarten' oder ,abwarten' glossiert wird.

Der Titel, Charaktere des Erfolgs' wurde in Bezug auf den (Verbal-)Charakter, also auf die inhärente aspektuelle Bedeutung gewählt (s.u.). Das Ziel der vorliegenden Studie ist es, Erfolg als ein Phänomen aus dem Bereich der Aspektualität systematisch zu beschreiben. Dazu muss man zunächst die Terminologie kartographieren.

\section{Basisterminologie}

Am Anfang jeglicher Untersuchung der Aspektualität steht die große Herausforderung, die Terminologie zu entflechten. Die seit mehr als hundert Jahren immer wieder gestellte Frage, ob es im Deutschen einen Aspekt gibt, stößt an das Problem der Definition der Basistermini. 


\section{Aspekt}

„Im Deutschen gibt es keinen Aspekt als grammatische Kategorie, wohl aber Aspektualität als funktional-semantische, konzeptuelle Kategorie, die sich auch im grammatischen System als verdeckte Kategorie, wenn auch peripher, auswirkt", meint ANDERSSON (2011: 10) und ich schließe mich dieser Meinung an. Mit Aspekt meine ich eine morphologische Kategorie, die den slawischen Sprachen eigen und bei jedem Verb ohne Kontext bestimmbar ist. Zugleich ist der Aspekt die morphologische Auswirkung einer semantischen Korrelation zwischen einem perfektiven und imperfektiven Partner.

Die zweite Gruppe der Definitionen versteht den Aspekt breiter, näher an der Definition der konzeptuellen Kategorie Aspektualität. BAUdOT (2004: 31) nennt zwei Aspekttypen: die Aktionsart und den syntaktischen Aspekt.

„Dieser Aspekt, manchmal als syntaktischer Aspekt genannt, ist eigentlich das Ergebnis des Zusammenwirkens sämtlicher syntaktischer Einheiten in der Äußerung." (BAudot 2004: 34)

Das Problem ähnlicher Definitionen liegt darin, dass man sprachspezifisch vorgeht ohne Rücksicht auf die slawischen Sprachen. In einem universalen System der Terminologie müsste man dann einen neuen Terminus für die spezifisch slawische Kategorie einführen.

Die meisten Basisverben im Tschechischen sind imperfektiv, der Ausgangsaspekt ist von der Semantik des Verbs (vom Verbalcharakter) abhängig. Das aspektuelle System funktioniert in Paaren oder Korrelationsreihen (VesELÝ 2014: 7). Aus einem imperfektiven Basisverb kann ein perfektives Verb mittels Präfigierung gebildet werden wie mýt-umýt, waschen', čist-prérečsst ,(durch)lesen'. Die Wahl des Präfixes erfolgt nach der sog. Bedeutungssubsumption: Es wird genau jenes Präfix gewählt, dessen inhärente Bedeutung der Bedeutung des Basisverbs am nächsten steht. Beispielsweise enthält die Semantik des Verbs psát, schreiben' den Prozess der Übertragung von Wörtern ,auf' ein Papier, deswegen wird als perfektivierendes Präfix na- ,auf-' gewählt (vgl. KOMÁREK 1984). Einem perfektiven Verb kann ein imperfektiver Partner mittels Wechsel des stammbildenden Suffixes gegenübergestellt werden, z.B. koupitkupovat, kaufen'. Die Imperfektivierung erfolgt auch bei den präfigierten perfektiven Verben (vgl. VeSELÝ 2014: 12) wie rozplést-rozplétat, aufdröseln'. Das durch Subsumption gebildete Präfixverb hat dabei entweder keinen sekundär imperfektivierten Partner, z.B. čcsst-přeč́čst* precítat, lesen', oder das imperfektive Verb ist synonym mit dem Basisverb z.B. mýt-umýtumývat $=m y ́ t$, waschen'. Die Paare imperfektives Verb - präfigiertes perfektives Verb werden manchmal als ,unecht' bezeichnet: „Solch ein sogenanntes, leeres' Präfix [...] modifiziert die lexikalische Bedeutung des Simplex zwar am wenigsten, doch es modifiziert sie - meist zur resultativen Aktionsart hin“"(Gross 1974: 31).

\section{Aktionsart}

In den vorangehenden Absätzen wurden bereits die Termini Aktionsart und Verbalcharakter benutzt. Die Aktionsart ist ein traditioneller Begriff, ursprünglich von BRUGMANN (1904) in die Aspektforschung eingebracht. Er ersetzte mit diesem Terminus die ,Zeitart' von 
Curtius (1852), der wiederum vom Terminus ,Zeitraum' (Heyse 1838) ausgegangen war. Erst AGRELL (1908) trennte die Aktionsarten vom Aspekt und definiert sie als

„Bedeutungsfunktionen der Verbalkomposita (sowie einiger Simplizia und Suffixbildungen), die genauer ausdrücken, wie die Handlung vollbracht wird, die Art und Weise ihrer Ausführung markieren" (AGRELL 1908: 78).

Bereits in dieser Definition spielt die Verbalkomposition (Präfigierung) eine wichtige Rolle. TsCHIRner (1991) berücksichtigt in seiner Arbeit über Aktionsarten die Präfigierung nicht, ${ }^{3}$ er legt den Fokus auf die Syntax und spricht nicht von Aktionsarten der Verben, sondern von den Prädikaten (Syntagmen) in Anlehnung an LöTsCHer (vgl. 1974: 258).

Es sind zwei Auffassungen der Aktionsartdefinition zu unterscheiden. Entweder wird der inhärente Zeitbezug von Verben/Prädikaten/Prädikationen gemeint oder gerade die Merkmalsunterschiede der derivierten Verben im Vergleich zum Stammverb.

„Es ist [...] sinnvoll, den Begriff der Aktionsart für das Wortbildungsphänomen zu reservieren: Aktionsarten werden durch Verbalpräfixe gegenüber den Basisverben eingebracht, peripher auch durch das Suffix -(e)l(lächeln, streicheln)“, so die Grammatik der deutschen Sprache (ZIFONUN et al. 1997: 1861).

Die Aktionsart wird also entweder inhärent oder relational gedeutet. In Anlehnung an Dressler (1968: 69) und Leiss (1992: 36) schließe ich mich der relationalen Deutung an. In einem Zeitschema wachen-einschlafen-schlafen-erwachen-... sind m.E. die Verben erwachen und einschlafen nur deswegen als ingressiv zu bezeichnen, da sie aus den Basisverben wachen und schlafen deriviert worden sind und demnach die ingressive Aktionsart ausdrücken. Sie bezeichnen das Eintreten eines neuen Zustands, erst die sekundäre Bedeutungskomponente kann ein Ende des alten Zustands darstellen, also eine Beziehung wachen-einschlafen bzw. schlafen-erwachen (vgl. STEINITZ 1977: 89).

\section{Verbalcharakter}

Die inhärente nicht-relationale aspektuelle Komponente der Verbbedeutung nennt DrESSLER (1968: 69) Verbalcharakter, Lyons (1977: 706) unterscheidet Aspekt von ,aspectual character', ComRIE (1981: 41) spricht von ,inherent meanings' des Aspekts. Die Notwendigkeit einer Unterscheidung des inhärenten Verbalcharakters und der relationalen Aktionsart ist gerechtfertigt. Die erwähnten Verben einschlafen und erwachen sind ähnlich wie finden oder kommen punktuell, schlafen und wachen sind durativ, ohne dass mit diesen Termini eine Beziehung zwischen den einzelnen Verben ausgedrückt würde. Die Durativität und Punktualität sind also Verbalcharaktere.

3 Er weist jedoch auf die Tatsache hin, dass „wahrscheinlich reihenbildende Präfixe und Halbpräfixe die Aktionsart jeweils auf die gleiche oder zumindest eine ähnliche Art beeinflussen“ (TsCHIRNER 1991: 103), und sondert die Präfixverben aus seinem Korpus aus. 


\section{Aspektualität}

Analog zu den konzeptuellen Begriffen Temporalität und Modalität wird heutzutage in der Forschung der Begriff Aspektualität als eine semantisch-konzeptuelle Kategorie, die alle internen aspektuellen Verhältnisse in der Sprache umfasst, d.h. auch Aspekt, Verbalcharakter und Aktionsarten.

Ein Konkurrenzbegriff ist Aktionalität (vgl. FläMIg 1965, TsCHIRNER 1991, ANDERsson 1972). Die heutige Linguistik neigt zum Terminus Aspektualität und da ich die beiden Termini synonym verstehe, werde ich über Aktionalität nicht referieren.

\section{Primat der Semantik}

Wie STEINItz (vgl. 1977: 86, 90) in ihren Überlegungen anführt, sind viele Unklarheiten in der Forschung der Aspektualität der Tatsache zuzuschreiben, dass man sich auf die Oberflächenstruktur beschränkt. Die semantische Einheit nennt STEINITZ semantisches Prädikat (1977: 86), ich benutze den Terminus Prädikation. Prädikationen sind als Ausgangspunkt praktisch, wenn man sich nicht auf eine Sprache beschränkt, denn es gilt, dass „im Prinzip jede Sprache - wenn auch mit noch so umständlichen Umschreibungen - alles bezeichnen kann" (Heger 1963: 69). Beispielsweise ist der perfektive Aspekt im Tschechischen eine grammatische Auswirkung der semantischen Perfektivität. Das Deutsche muss das Verb in einen bestimmten lexikalischen Kontext setzen oder andere Mittel benutzen, um solche Prädikation ausdrücken zu können.

Der Verbalcharakter ist eine Eigenschaft des Verbs und daher sekundär auch der Prädikation, wenn sie nicht weitere Mittel der syntaktischen Oberfläche ändern (s.u.). Beispielsweise ist das Verb treffen punktuell. Die prototypische Prädikation ist demnach auch punktuell. Die Aktionsart stellt den oberflächlichen Ausdruck einer semantischen Relation dar. So kann z.B. die ingressive semantische Relation durch ingressive Aktionsart (erblühen zu blühen), Beziehung von zwei inhärenten Bedeutungen (erfahren zu wissen), kontextuelle Mittel (auf einmal hat sie gesprochen) oder spezifische syntaktische Mittel (Konstruktionen mit werden, in + verbales Substantiv + begriffen sein usw.) ausgedrückt werden (vgl. ŠTíCHA 2003: 582).

\section{Klassifikation der Verben}

Zunächst stellt sich die Frage, was soll eigentlich klassifiziert werden: Prädikationen, syntaktische Prädikate oder Verben? Die Gruppe der (semantischen) Prädikationen ist sehr abstrakt, manche Prädikationen können in keiner der behandelten Sprachen morphologisch oder syntaktisch ausgedrückt werden. Eine vollständige universelle Kategorisierung aller möglichen Prädikationstypen nach der internen temporalen Zeitstruktur in allen Sprachen der Welt ist für die Zwecke dieser Studie nicht nötig. 
Den Vorteil des syntaktischen Ansatzes von LöTscher (1974) und Tschirner (1991), die Prädikationen nach der Aspektualität (nach dem Charakter) klassifizieren, ${ }^{4}$ zeigen die Testverfahren. Im Deutschen spielt der syntaktische Kontext eine umso größere Rolle, weil es keinen paradigmatischen Aspekt gibt. Beispielsweise ändert das Akkusativobjekt bei den Verben, die sowohl transitiv als auch intransitiv gebraucht werden können, die Aspektualität (den Charakter) des Satzes (vgl. Comrie 1981: 44).

(5) Er singt. Er hat gesungen.

(6) Er singt ein Lied. Er hat ein Lied gesungen.

(7) Er singt Lieder. Er hat Lieder gesungen.

Im Beispiel (5) handelt es sich um eine atelische/dynamisch-durative ${ }^{5}$ Prädikation, in (6) um eine telische/terminative, in (7) ist die Klassifizierung umstritten. In (6) gibt es einen Anfang und ein Ende der Prädikation, in (5) nicht. Nach einer semantischen Analyse ist es jedoch klar, dass der Anfang und das Ende die Grenzen des Liedes sind, in diese Grenzen wird das durative Verb singen, eingegossen'. Ähnlich z.B. bei eine Zigarette rauchen, wo das Ende der Zigarette das Ende des sonst durativen Rauchens bestimmt.

Das Tschechische kann die Sätze (5-7) folgendermaßen übersetzen:

(5a) Zpívá. Zpíval. / (5b) Zazpívá. Zazpíval.

(6a) Zpívá písničku. Zpíval písničku. / (6b) Zazpívá písničku. Zazpíval písničku.

(7a) Zpívá písničky. Zpíval písničky. / (7b) Zazpívá písničky. Zazpíval písničky.

Die ersten Sätze in (5b, 6b und 7b) sind perfektiv und daher zukünftig, die deutschen Sätze im Präsens können mit einer Angabe wie morgen auch zukünftig sein. Ist eine Prädikation wie in er singt morgen noch immer atelisch/durativ? Solche kontextuelle Mittel können im Deutschen die Aspektualität ändern. Die durative Prädikation in 6a kann im Deutschen oberflächlich durch ein Inzidenzschema (vgl. Gross 1974: 71-72) wie in (6c) ausgedrückt werden:

(6c) Er hat ein Lied gesungen, als er plötzlich die Stimme verloren hat.

Obwohl die kontextuellen Mittel mit der Bedeutung des syntaktischen Prädikats spielen können, ist der inhärente Verbalcharakter bei Verben wie singen oder rauchen durativ. Aus diesen Gründen klassifiziere ich jetzt semantisch die tschechischen und deutschen Verben, nicht Prädikate oder Prädikationen. Die primäre Opposition ist dabei die zwischen den durativen und terminativen Verben.

4 Sie klassifizieren primär die Oberfläche, d.h. syntaktische Prädikate, jedoch nach der Tiefenstruktur, nach den inhärenten aspektuellen Bedeutungen der ganzen Aussagen. Man könnte den Begriff, Charakter der Prädikation' benutzen, denn es handelt sich um die inhärente Bedeutung, nur nicht auf der Ebene des morphologischen Verbs wie der Verbalcharakter, sondern auf der Ebene der ganzen Prädikation.

5 Das erste Glied folgt der Klassifizierung von Comrie (1981), das zweite von TsChirner (1991). 


\section{Durative Verben}

Die durativen Verben ,dauern', sie haben keinen Anfang oder Ende, es handelt sich im Deutschen in der Regel um Basisverben, im Tschechischen besitzen fast alle imperfektiven Verben das semantische Merkmal durativ. ${ }^{6}$ Sie sind zu jedem Zeitpunkt gültig (vgl. SACKer 1988: 213), das Testverfahren von VendLer (1957: 100) lautet mit leichter Veränderung: ,Wenn ich aufhöre zu X, habe ich ge-X-t?' wobei die morphologischen Konstruktionen selbstverständlich veränderlich sind. Diese Gruppe enthält (A) Zustände, states, statisch-durative Verben wie stehen, liegen, bleiben, sein, existieren und (B) durative Handlungen, Vorgänge, dynamisch-durative Verben wie laufen, gehen, singen, reden, suchen.

Die Grenze zwischen den Zuständen und den Vorgängen ist fließend: Tschirner (vgl. 1991: 106) spricht über Energie, die vom Agens ausgegeben wird, und erwähnt ein Testverfahren mit aufhören $z u$, das die Unterschiede nicht deutlicher macht. Beispiele wie aufhören zu bleiben sind unlogisch, jedoch zählt man dieses Verb zu den Zuständen. Die tschechische ,akademische‘ Grammatik (Petr 1986) nennt Zustände und einfache Vorgänge als Ausnahmen bei den Aspektkorrelationen. Durative Verben wie stát stehen', pracovat ,arbeiten', svitit , leuchten' u.a. haben keine perfektiven Aspektpartner. Verben wie císt , lesen' sind ohne Ergänzung auch nicht perfektivierbar:

(8) Četl jsem. *Přečetl jsem., Ich las.

Es ist des Weiteren für die Suche nach dem Erfolg wichtig, das Merkmal ,zielgerichtet ' bei einigen durativen Verben zu beobachten:

(9) Wenn ich aufhöre zu warten, habe ich gewartet?

(10) Wenn ich aufhöre zu suchen, habe ich gesucht?

(11) Wenn ich aufhöre zu laufen, bin ich gelaufen?

Alle Fragen sind mit ja zu beantworten, alle drei Verben sind durativ. Beim folgenden Testverfahren sieht man allerdings einen feinen Unterschied:

(9a) Wenn ich aufhöre zu warten, war das Warten erfolgreich?

(10a) Wenn ich aufhöre zu suchen, war das Suchen erfolgreich?

(11a) Wenn ich aufhöre zu laufen, war das Laufen erfolgreich?

In (9 und 10) ist der Erfolg an das konkrete Ziel gebunden, die Verben warten und suchen sind zielgerichtet. Das Verb laufen trägt dieses Merkmal nicht inhärent.

\section{Terminative Verben}

Terminative Verben gelten „erst mit ihrem tatsächlichen Abschluss als realisiert“" (SACKER 1988: 213). Die tschechischen perfektiven Verben sind immer terminativ, denn sie sind als Ganzes perspektiviert, inkl. des Beginns und des Abschlusses. Die erste Gruppe der

\footnotetext{
6 Nur sekundäre Imperfektivierungen von punktuellen Verben sind iterativ, nicht durativ, vgl. d.W.
} 
terminativen Verben sind die punktuellen Verben. Der Beginn und der Abschluss der Prädikation fallen zusammen. Im Tschechischen ist der imperfektivierte Partner ausschließlich iterativ zu deuten wie prijiit-pricházet ,kommen'. VENDLER (1967: 103) gebraucht für die punktuellen Verben die Bezeichnung ,achievements“. Die nicht-punktuellen terminativen Verben sind telisch, SACKER nennt sie zyklische Handlungen (1983: 213), VENDLER „accomplishments" (1967: 107). In diese Gruppe gehören z.B. die Verben überreden oder verteidigen. Sie sind immer zielgerichtet, nicht punktuell und nicht zu jedem Zeitpunkt gültig, die Ergebnisse der Proben (12 und 13) sind negativ.

(12) Wenn ich aufhöre, ihn zu überreden, habe ich ihn überredet?

(13) Wenn ich aufhöre, die Arbeit zu verteidigen, habe ich sie verteidigt?

\section{Erfolgsähnliche Aktionsarten}

Eine vollständige Kategorisierung aller Aktionsarten ist für die Zwecke der vorliegenden Arbeit nicht nötig. Bevor ich aber zu der Erfolgskategorie komme, möchte ich kurz über diejenigen Aktionsarten referieren, die sich auf die Endphase der Basisverb-Prädikation beziehen.

\section{Egressive Aktionsart}

Die egressive Aktionsart bezeichnet die Endphase eines durativen Basisverbs, im Deutschen z.B. verblühen (zu blühen), ausklingen (zu klingen). Das Basisverb ist nicht zielgerichtet, daher hat die Egressivität mit dem Erfolgskonzept nichts zu tun. Die semantische Relation, die zwischen den Verben entsteht und durch die Aktionsart realisiert wird, ist das Ende eines Vorgangs/Zustands und der Anfang eines anderen (Null-)Zustands. Die Beziehung zwischen den Bedeutungen von leben-sterben gehört auch zu den egressiven semantischen Relationen.

Im Tschechischen wird die egressive Aktionsart oft mit dem Präfix do- gebildet. Die Paare doznit-znit , ausklingen-klingen', domluvit-mluvit ,aufhören zu sprechen-sprechen' sind keine Aspektpaare, denn die Bedeutung der Derivate enthält keinen Anfang des Klingens bzw. Sprechens. Die Imperfektivierungen doznivat und domlouvat sind nicht mit znit und mluvit synonym.

\section{Resultative/Perfektive Aktionsart}

SACKer (1988: 233) spricht von Resultativität im Satz (14) im Gegensatz zu Perfektivität im Satz 15), die den resultativen Zustand präsupponiert. Wiederum sind aber diese Überlegungen syntaktisch-semantischer Natur.

(14) Sie ist schon lange tot.

(15) Sie ist gestorben. 
Steinitz (1977: 90) sieht in allen diesen Beziehungen die Inchoativität. Die SterbenPrädikation sei ein Übergang zwischen zwei antonymischen Zuständen: von leben zu tot sein.

Ich halte die Termini „resultative Aktionsart" und „perfektive Aktionsart" für synonym. Die resultative/perfektive Aktionsart wird mit Beispielen wie aufessen (zu essen), erjagen (zu jagen) im Deutschen belegt. Das letztgenannte Paar (vgl. Gallmeier 2005: 2) ist aber anders als die ersten beiden. Die Erjagen-Prädikation resultiert zwar in erjagt sein, sie enthält aber nicht den ganzen Prozess des Jagens im Gegensatz zu aufessen, das perfektiv zu deuten ist. Die perfektive Aktionsart ist im Tschechischen als Aspekt grammatikalisiert, umstritten können nur die erwähnten ,unechten' Aspektpaare sein wie mýt-umýt ,waschen', jist-snist ,essen-aufessen'.

\section{Erfolg}

Erfolg ist eine Relation. Ein punktuelles Verb repräsentiert eine Prädikation, die eine vorausgenommene zielgerichtete Prädikation präsupponiert und erfolgreich abschließt. Es geht lediglich um Präsupposition, nicht um eine obligatorische Bedeutungskomponente. Demnach können auch Kontexte auftreten, wo die zielgerichtete Prädikation nicht vorangeht wie der in der Einführung erwähnte Satz (2):

(2) Ich habe auf der Straße eine Hundertnote gefunden.

Solcher Kontext ist aber semantisch komplex und bedürfte noch weiterer semantischen Analyse. Hypothetisch kann man z.B. die Kollokation auf der Straße finden nicht mit beliebigen Objekten auf der Straße verbinden, sondern nur mit solchen, die man üblicherweise verliert, sucht oder allgemein haben will (Hundertnote, Knopf, 4-blättriges Kleeblatt, aber nicht Pflasterstein, Gras, Straßenbeleuchtung).

Es kommen zwei Möglichkeiten des Erfolgs-Ausdrucks auf der verbalen Ebene in Frage: (A) die zielgerichtete Prädikation kommt durch ein telisches Verb zum Ausdruck; (B) die zielgerichtete Prädikation kommt durch ein duratives Verb zum Ausdruck.

$\operatorname{Ad}(\mathrm{A})$ : Bei den deutschen telischen Verben wird die Erfolgs-Relation kontextuell erbaut. Die Temporalität spielt eine wichtige Rolle: ${ }^{7}$

(16) Sie hat die Arbeit verteidigt. (+) Sie hat die Arbeit zwei Stunden lang verteidigt. (-)

(17) Sie verteidigt (gerade) die Arbeit. (-)

(18) Sie verteidigt die Arbeit (morgen), ich bin mir sicher. (+)

Im Tschechischen ist der Aspekt das entscheidende Kriterium bei diesen Verben:

(16a) Obhájila práci. (+) Obhajovala práci. (-)

(17a) Obhajuje práci. $(-)$

(18a) Zítra práci obhájí. (+) Zítra práci bude obhajovat (-)

7 (+) bezeichnet die Anwesenheit und (-) die Abwesenheit der Erfolgskomponente in der Prädikation. 
Ad (B): Durative zielgerichtete Verben implizieren eine Möglichkeit des Erfolgs, nicht immer ist es allerdings möglich, den Erfolg auszudrücken. Die einfachste Möglichkeit dafür ist die sukzessive Aktionsart. Im Deutschen ist das erwähnte Paar jagen-erjagen ein Beispiel dafür. Jagen ist ein durativer zielgerichteter Vorgang, das Ziel des Jagens ist das punktuelle accomplishment - das Erjagen. Das Verb erjagen ist punktuell und präsupponiert einen vorangegangenen zielgerichteten Prozess, obwohl theoretisch nicht gejagt worden sein muss. Im Tschechischen werden die Verben lovit,jagen' und ulovit, erjagen, fangen' manchmal fälschlich als Aspektpaar interpretiert. Das Verb lovit ist ein einfacher Prozess und hat keinen Aspektpartner, ulovit schließt nicht den ganzen Prozess des Jagens ein, das Präfix $u$ - ist nicht rein perfektivierend. ${ }^{8}$

Im Bereich der sukzessiven Aktionsarten ist das Tschechische reicher als das Deutsche, es gibt nämlich die Gruppe der reflexiven Derivate mit do-, die zu den zielgerichteten Vorgängen den sukzessiven Partner darstellen:

(19) Ich habe gewartet. (-)

(20) Ich habe ihn angerufen. (-)

(19a) Čekal jsem. (-) (19b) Dočkal jsem se. (+)

(20a) Volal jsem mu. (-) (20b) Dovolal jsem se mu. (+)

Der Satz (19b) bedeutet, dass ich auf etwas (ein Ziel) gewartet habe, das realisiert worden ist; (20b), dass er den Hörer abgenommen hat.

Die beiden Sprachen verfügen auch über Basisverben, die in einer Erfolgs-Relation mit anderen Verben stehen, typischerweise suchen-finden, jagen-fangen, spielen-gewinnen. Das letztgenannte Paar kommt im Tschechischen auch durch die Derivation hrát-vyhrát zum Ausdruck.

\section{Zusammenfassung}

Die vorliegende Studie hat versucht, ein System der Aspektualität im Tschechischen und Deutschen zu skizzieren und in dieses System die Erfolgskategorie einzusetzen. Es sind zunächst die wichtigsten Basistermini definiert worden: Aspekt als eine den slawischen Sprachen eigene morphologische Kategorie, die sowohl inhärent als Bedeutungskomponente jedes Verbs zu bestimmen als auch relational als grammatikalisierte Auswirkung der semantischen Korrelation zwischen einer imperfektiven und perfektiven Perspektivierung einer Prädikation ist; Aktionsart als ein Ausdruck der semantischen Relation zwischen einem Basisverb und seinem Derivat; Verbalcharakter als eine inhärente Komponente der Verbbedeutung, entweder durative oder terminative. Durative Verben können Zustände und Vorgänge bezeichnen.

8 Manchmal wird das Verb doch als perfektiver Partner des impf. Prozesses benutzt wie z.B. in Jdu nèco ulovit. Jdu to najit. ,Ich gehe etwas fangen. Ich gehe das finden'. Solche Sätze sind synonym mit Jdu lovit. Jdu to bledat. ,Ich gehe jagen. Ich gehe das suchen' Weil sowohl lovit als auch bledat zielgerichtet sind, ist der Gebrauch der sukzessiven Aktionsart eigentlich redundant und nur stilistisch bedingt. Solche Kontexte bedürfen aber noch weiterer Analysen. 
Die Vorgänge können zielgerichtet sein. Terminative Verben sind entweder punktuell oder telisch, die telischen Verben sind immer zielgerichtet. Ohne das Erreichen des Ziels gilt das Verb als nicht realisiert, z.B. bestellen, verteidigen, überzeugen.

Die Erfolgs-Relation kommt nur bei den zielgerichteten Verben in Frage. Erstens bei telischen Verben, die im Deutschen kontextuell entweder als erfolgreich realisiert oder nicht realisiert wie in den Beispielen (16-18) gelten. Im Tschechischen übernimmt der Aspekt die Rolle des Kontextes. Der perfektive Aspekt macht das telische Verb erfolgreich realisiert. Zweitens kommt Erfolg als Relation zwischen einem durativen zielgerichteten Vorgang und dem punktuellen Erreichen des Ziels zum Ausdruck. Das Ziel wird entweder durch ein punktuelles Basisverb (finden zu suchen) oder als sukzessive Aktionsart (erjagen zu jagen, vyhrát ,gewinnen' zu hrát, spielen', dockkat se zu čekat, warten' usw.) repräsentiert. Selbstverständlich können auch kontextuelle Mittel, Umschreibungen usw. die semantische Relation zum Ausdruck bringen. Wichtig werden diese Überlegungen bei der Untersuchung von Übersetzungen aus dem Tschechischen ins Deutsche sein, wo ich bereits viele Fehler z.B. bei der Übertragung sukzessiver Aktionsarten beobachtet habe. Eine Analyse der Übersetzungen wird ein Teil meiner vorgesehenen Dissertation werden.

\section{Bibliographie}

Agrell, Sigurd (1908): Aspektänderung und Aktionsartbildung beim polnischen Zeitwort. Lund: Håkan Ohlssons Buchdruckerei.

Andersson, Sven-Gunnar (2011): Gibt es Aspekt im Deutschen? In: Gautier, Laurent / HaberKORN, Didier (Hg.): Aspekt und Aktionsarten im heutigen Deutsch. Tübingen: Stauffenburg, 1-11.

Andersson, Sven-Gunnar (1972): Aktionalität im Deutschen. Eine Untersuchung unter Vergleich mit dem russischen Aspektsystem. I: Die Kategorien Aspekt und Aktionsart im Russischen und Deutschen. Uppsala: Acta Universitatis Upsaliensis.

BAUDot, Daniel (2004): Aspekt und Aspektualität: kleiner Beitrag zur Klärung von Begriffen. In: GAUTIER, Laurent / HABERKoRn, Didier (Hg.): Aspekt und Aktionsarten im heutigen Deutsch. Tübingen: Stauffenburg, 31-42.

BRU GM Ann, Karl (1904): Kurze vergleichende Grammatik der indogermanischen Sprachen. Straßburg: Karl Trübner.

Comrie, Bernard (1981): Aspect. An Introduction to the Study of Verbal Aspect and Related Problems. 2. Aufl., Cambridge: Cambridge University Press.

CuRTIUs, Georg (1852): Griechische Schulgrammatik. Prag: Verlag der J. G. Calveschen Buchhandlung. Dessì SCHMID, Sarah (2014): Aspektualität. Ein onomasiologisches Modell am Beispiel der romanischen Sprachen. Berlin; Boston: de Gruyter.

Dressler, Wolfgang (1968): Studien zurverbalen Pluralität. Iterativum, Distributivum, Durativum, Intensivum in der allgemeinen Grammatik, im Lateinischen und Hethitischen. Wien: Verlag der Österreichischen Akademie der Wissenschaften.

FLäMIG, Walter (1965): Zur Funktion des Verbs. III. Aktionsart und Aktionalität. In: Deutsch als Fremdsprache 2, 4-12. 
Gallmeier, Maria (2005): Wo liegt die Grenze? Aspekt, Aktionsart und verwandte Phänomene in der Erforschung der deutschen Sprache. Knoxville: University of Tennessee.

Gross, Harro (1974): Der Ausdruck des,Verbalaspekts' in der deutschen Gegenwartssprache. Hamburg: Helmut Buske.

Heger, Klaus (1963): Die Bezeichnung temporal-deiktischer Begriffskategorien im französischen und spanischen Konjugationssystem. Tübingen: Niemeyer.

Heyse, Johann Christian August (1838): Theoretisch-praktische deutsche Grammatik oder Lebrbuch zum reinen und richtigen Sprechen, Lesen und Schreiben der deutschen Sprache. Neu als Ausführliches Lebrbuch der deutschen Sprache, bearbeitet von K.W.L. Heyse, Bd. 1, Hannover: Hahnsche Hofbuchhandlung.

KomÁReK, Miroslav (1984): Prefixace a slovesný vid (K prefixům prostě vidovým a subsumpci) [Präfigierung und der verbale Aspekt (zu den rein aspektuellen Präfixen und Subsumption)]. In: Slovo a slovesnost 45, 257-267.

LeIss, Elisabeth (1992): Die Verbalkategorien des Deutschen. Berlin; New York: de Gruyter.

Lötscher, Andreas (1974): Satzsemantik und Zeitschemata. Sprachsystem und Sprachgebrauch. In: ENGel, Ulrich / Grebe, Paul (Hg.): Festschrift für Hugo Moser. Teil 1, Düsseldorf: Schwann, $248-272$.

Lyons, John (1977): Semantics. Bd. 2. Cambridge: Cambridge University Press.

Petr, Jan (Hg.) (1986): Mluvnice čestiny 2. Tvarosloví [Grammatik des Tschechischen 2. Morphologie]. Prag: Academia.

SACKER, Ulrich (1983): Aspektueller und resultativer Verbalausdruck im Französischen, Italienischen, Russischen und Deutschen. Tübingen: Narr.

Schopf, Alfred (1984): Das Verzeitungssystem des Englischen und seine Textfunktion. Tübingen: Niemeyer.

STEINITZ, Renate (1977): Zur Semantik und Syntax durativer, inchoativer und kausativer Verben. In: Linguistische Studien A. H. 35, 86-129.

ŠTícHA, František (2003): Česko-némecká srovnávací gramatika [Tschechisch-deutsche vergleichende Grammatik]. Prag: Argo.

TICHÁK, Viktor (2018): Konstitution des Aspektualitätsgedankens. In: HorŇÁČEK, Milan / KRAPPMANN, Jörg / Rinas, Karsten (Hg.): Vom Nutzen diskursanalytischer Verfahren, Olomouc. Univerzita Palackého v Olomouci, 59-79.

TsCHIRner, Erwin (1991): Aktionalitätsklassen im Neuhochdeutschen. New York: Lang.

Vendler, Zeno (1967): Verbs and Times. In: Linguistics in Philosophy. Ithaca: Cornell University Press, 97-121.

VESELÝ, Luboš (2014): Gramatické studie I. Př́spěvky k české aspektologii [Grammatische Studien I. Beiträge zur tschechischen Aspektologie]. Olomouc: Univerzita Palackého v Olomouci.

Zifonun, Gisela et al. (1997): Grammatik der deutschen Sprache. Berlin; New York: de Gruyter. 